mortality. Epigenetic changes might play important roles in mediating chronic fibroblast activation. Trimethylation of $\mathrm{H} 3$ at lysine residue K27 (H3K27me3) is a repressive epigenetic mark that was recently identified as an important negative regulator of fibroblast activation [1]. Jumonji domain containing protein 3 (JMJD3) mediates H3K27me3-demethylation. JMJD3 inhibitors are being tested as therapeutic strategies in malignant diseases.

Objectives: The aim of this study was to characterize the role of JMJD3 in fibrotic disease and to explore JMJD3 as a potential drug target in SSc.

Methods: Expression analyses of JMJD3 were performed using qPCR, IF and Western blot. siRNA mediated knockdown and the pharmacologic H3K27me3demethylase inhibitor GSKJ4 were used to target JMJD3. In vivo, we analyzed the effects of GSKJ4 in bleomycin-induced dermal fibrosis and in TopoisomeraseI-induced (Topol) fibrosis. H3K27me3 levels at the Fra2 promotor were analyzed by CHIP.

Results: We observed increased expression of JMJD3 in SSc skin compared to healthy controls. Fibroblast-specific overexpression of JMJD3 was also reflected in experimental fibrosis models. TGF $\beta$ upregulated JMJD3. Inhibition of JMJD3 increased $\mathrm{H} 3 \mathrm{~K} 27 \mathrm{me} 3$ in vitro and in vivo. Inhibition of JMJD3 reverted the activated fibroblast phenotype in SSc fibroblasts and decreased the expression of contractile fibers and of $\alpha$-smooth muscle actin. In addition, JMJD3 inhibition reduced the basal and TGF $\beta$ induced collagen secretion of SSc fibroblasts. JMJD3 regulated the TGF $\beta$ induced expression of Fra2. GSKJ4 reverted the TGF $\beta$ induced reduction of $\mathrm{H} 3 \mathrm{~K} 27 \mathrm{me} 3$ at the Fra2 promotor. Moreover, the anti-fibrotic effects of JMJD3 inhibition were evened in Fra2 knockout fibroblasts. Overexpression of Fra2 in JMJD3-knockdown fibroblasts restored the profibrotic effect of JMJD3. In vivo, inhibition of JMJD3 ameliorated fibrosis in bleomycin- and Topol- induced experimental fibrosis and reduced dermal thickening, hydroxyproline content and myofibroblast differentiation.

Conclusions: We present first evidence that JMJD3 contributes to the activated phenotype of SSc fibroblasts. TGF $\beta$ upregulated JMJD3. Inhibition of JMJD3 prevented the aberrant activation of fibroblasts in vitro and ameliorated dermal fibrosis in several mouse models in vivo. The profibrotic effects of JMJD3 might be mediated by reducing the $\mathrm{H} 3 \mathrm{~K} 27 \mathrm{me} 3$ at the Fra2 promotor and consecutive overexpression of Fra2

References:

[1] Kramer, M., et al., Inhibition of H3K27 histone trimethylation activates fibroblasts and induces fibrosis. Ann Rheum Dis, 2013. 72(4): p. 614-20.

Disclosure of Interest: None declared

DOI: 10.1136/annrheumdis-2017-eular.3792

\section{SAT0319 MULTIPARAMETRIC DETECTION OF AUTOANTIBODIES TO INVESTIGATE RELATIONSHIPS BETWEEN SEROLOGICAL AND CLINICAL SUBSETS OF SYSTEMIC SCLEROSIS PATIENTS}

P. Budde ${ }^{1}$, H.-D. Zucht ${ }^{1}$, D.S. Wirtz ${ }^{1}$, K. Marquart ${ }^{1}$, M. Schneider ${ }^{2}$ P. Schulz-Knappe ${ }^{1}$, N. Hunzelmann ${ }^{3} .{ }^{1}$ Protagen Ag, Dortmund; ${ }^{2}$ Rheumatology \& Hiller-Forschungszentrum, Heinrich-Heine-University, Düsseldorf;

${ }^{3}$ Dermatology, University of Cologne, Cologne, Germany

Background: Systemic sclerosis (SSc) is a largely heterogeneous autoimmune disease, with patients exhibiting an extensive range of clinical presentations and various disease course. The most widely used classification divides SSc into two major subsets diffuse cutaneous (dcSSc) and limited (IcSSc) SSc by the extent and severity of skin fibrosis. However, not all patients fit into these subsets. This has created great interest to examine disease heterogeneity at the molecular level to uncover unrecognized SSc subtypes that may differ with regard to clinical manifestations, prognosis or therapy response.

Objectives: In large-scale "omics"-type autoantibody (AAB) profiling studies we have recently identified novel SSc-associated autoantigens. Here, we describe the development of a 20 marker multiplexed AAB assay to facilitate the discovery and validation of $A A B$-based patient subgroups.

Methods: A Luminex bead-based AAB assay was designed by combining 8 connective tissue disease (anti-centromere, anti-Scl70, U1-snRNP, SSB, Ro52, Ro60, SmB, anti-ribosomal P) antigens with 12 novel antigens (including BICD2, JMJD3/KDM6B, and PPP1R2). Novel AAB targets were previously detected in SSc patients with a p-value $<0.05$ (Mann-Whitney-U-test) and frequency $>15 \%$. AAB reactivity was analysed in 92 SSc patients (dcSSc: $n=32$, IcSSc: $n=50, S S c$ overlap: $n=9$ ). The mean modified Rodnan skin score (MRSS), mean disease duration (month), and mean age (years) of the SSc cohort was 10.51, 162.5 and 56.94, respectively. To analyze the individual-level patient similarity of $A A B$ reactivity, the total number of $A A B s$ reactive in each patient was calculated and referenced to the number of all available antigens in percent. Patient's demographics and clinical data were dichotomized into patients with mRSS higher or lower than the mean value. Hierarchical cluster analysis was performed to investigate the relationship between $A A B$ patient signatures and clinical and demographic features.

Results: Based on their AAB reactivity pattern, the SSc sample cohort can be decomposed into four apparent clusters and additional fine-level clusters. LcSSc patients were spread over three clusters, each with clearly distinct AAB profile. Compared to dcSSc patients, IcSSc patients were more heterogeneous in their AAB profile. The percentage of IcSSc patients in clusters $1-3$ was $70 \%, 90 \%$ and $53 \%$, respectively. Patients in cluster 2 had an extended $A A B$ repertoire that is anti-centromere, KDM6B, SSA, BICD2 and PPP1R2. $63 \%$ of all patients in cluster 4 were dcSSc and anti-Scl70 positive, who were most afflicted of the disease. $A A B$ signatures of patients were mapped against dichotomized demographic and clinical features. Moving from cluster 1 to 4 the number of patients with shorter disease duration, lower age, higher mRSS and higher frequency of lung involvement increased.

Conclusions: The multiplexed analysis of AABs in SSc enables defining an $A A B$ reactivity score and patient clusters. This might support to subclassify SSC beyond ICSSc and dSSc.

Disclosure of Interest: P. Budde Employee of: Protagen AG, H.-D. Zucht Employee of: Protagen AG, D. Wirtz Employee of: Protagen AG, K. Marquart Employee of: Protagen AG, M. Schneider Consultant for: Protagen AG, P. Schulz-Knappe Employee of: Protagen AG, N. Hunzelmann: None declared DOI: 10.1136/annrheumdis-2017-eular.1982

\section{SAT0320 SSC- IGG EFFECTS ARE MEDIATED THROUGH DISTINCT PATHWAYS IN THP-1 CELLS}

G. Eleftheriadis ${ }^{1}$, M. Wannick ${ }^{1}$, C. Sadik ${ }^{1}$, G. Riemekasten ${ }^{2}{ }^{1}$ Institute of Experimental Dermatology; ${ }^{2}$ Department of Rheumatology, University of Lübeck, Lübeck, Germany

Background: Peripheral blood mononuclear cells (PBMCs) are thought to play a key role in the pathogenesis and progress of systemic scleroderma (SSc) with patients displaying distinct shifts in count, receptor expression profile and cytokine secretion patterns. SSc-lgG with elevated anti-AT ${ }_{1} R$ (angiotensin II type 1 receptor)/ $\mathrm{ET}_{\mathrm{A}} \mathrm{R}$ (endothelin-1 type $\mathrm{A}$ receptor) - $\mathrm{AAb}$ (autoantibody) titers have been correlated to disease severity and progression ${ }^{1}$. It remains poorly understood through which pathways SSc- IgG mediates its effects.

Objectives: In this study we sought to analyze the expression patterns of THP-1 cells (a monocytic cell line) after SSc-IgG application and their reversibility through application of numerous pharmacological inhibitors.

Methods: Transcription of IL-8- and CCL-18 in THP-1-cells after SSc-lgG and normal IgG stimulation was quantified by QPCR. Stimulations of THP- 1 cells with total IgG of phenotypically different groups of SSc patients as well as ET-1 and AT-2 were carried out. In addition, stimulation with pharmacological inhibitors was conducted in a dose-dependent-manner. The results were quantified by IL-8- and CCL18-ELISA of the supernatants.

Results: Expression of IL-8 and CCL-18 is induced by SSc-lgG treatment in comparison to normal lgG which does not follow this trend. IL-8 secretion of THP-1 cells upon SSc-lgG stimulation is mediated through specific autoantibody effects and transduced through NF-KB, ERK-, and AP-1 pathways. CCL-18 secretion of THP-1 cells upon SSc-lgG stimulation is not mediated through aforementioned pathways.

Conclusions: A stable cell culture system able to reproduce previous PBMC data on IL-8 and CCL-18 induction upon SSc-lgG-treatment could be established and insight was gained regarding key pathways which are involved in the transduction leading to IL-8 secretion. The effects of specific surface receptor expression profiles on transduction remain to be elucidated.

\section{References:}

[1] Günther, J., Rademacher, J., van Laar, J.M. et al. Semin Immunopathol (2015) 37: 529 .

Disclosure of Interest: None declared

DOI: 10.1136/annrheumdis-2017-eular.5314

\section{SAT0321 MKP-1 AS A PROTECTIVE FACTOR AND NOVEL DRUG TARGET IN SCLERODERMA: MKP-1 DEFICIENT MICE DEVELOP MORE SEVERE DERMAL FIBROSIS IN A WIDELY USED EXPERIMENTAL MODEL OF SCLERODERMA}

M. Scotece, M. Hämäläinen, T. Leppänen, E. Moilanen. The Immunopharmacology Research Group, Faculty of Medicine and Life Sciences, University of Tampere and Tampere University Hospital, Tampere, Finland

Background: Scleroderma is a chronic connective tissue disease of unknown aetiology. In early stages, vascular injury and inflammation lead to fibrosis, resulting in irreversible damage in various organs. Inflammation is believed to be necessary in order to activate fibroblasts to over-produce extracellular matrix components. At the present, there is no effective standard treatment to reverse or slow down the progression of scleroderma but one of the feasible approaches is to target key inflammatory pathways that are involved in the pathogenesis of the disease.

MKP-1 (Mitogen-Activated Protein Kinase Phosphatase-1) is a nuclear phosphatase present in most cell types and tissues. Studies with MKP-1 deficient mice have undoubtedly shown that MKP-1 is an important regulator of innate and adaptive immune responses to limit and suppress inflammation (1) but its role in fibrosing diseases has not been studied.

Objectives: In the present study, we aimed to investigate the potential protective role of MKP- 1 in the pathogenesis of scleroderma by using MKP-1 deficient mice and a widely studied experimental model of scleroderma.

Methods: We used bleomycin-induced dermal fibrosis in the mouse as an experimental model of scleroderma (2). Wild type (WT) and MKP-1 deficient mice were injected subcutaneously with bleomycin every other day for 28 days. Dermal 